\title{
Comment on "Evaluation of serum interleukin-6 levels in hepatocellular carcinoma patients: a systematic review and meta-analysis"
}

\author{
Mattia Bellan, Gian Carlo Avanzi, Mario Pirisi \\ Università del Piemonte Orientale, UPO, Italy
}

Address for correspondence

Dr. Mattia Bellan, Università del Piemonte Orientale, UPO, Italy, e-mail: mattia.bellan@med.uniupo.it

\section{Dear Editor}

We read with interest the recent meta-analysis by Shakiba et al. [1], which demonstrated that serum interleukin 6 (IL-6) levels are significantly higher in patients affected by hepatocellular carcinoma (HCC) compared to healthy controls and to patients with cirrhosis or chronic hepatitis. The authors conclude that IL-6 might play a pathogenetic role in hepatocarcinogenesis and that serum IL-6 may represent a potentially useful diagnostic biomarker. We agree with these conclusions, but we argue that a significant advance in understanding the relationship between serum IL-6 and HCC will require refining the analysis by taking into account factors that Shakiba et al. could not consider, the most important of which - at least in our opinion - is gender. In fact, HCC is characterized by a significant gender disparity, due to multiple mechanisms among which sex differences in MyD88-dependent IL- 6 production have been considered paramount [2]. Experimental hepatocarcinogenesis data show that the administration of diethylnitrosamine (DEN) causes a larger increase in serum IL-6 in male mice than it does in female mice. Genetic ablation of IL- 6 abolishes the gender differences in liver injury and the administration of estrogens to male DEN-treated mice decreases serum IL-6 concentration [2]. Estrogen-mediated inhibition of IL- 6 production by Kupffer cells may thus protect females from developing liver cancer, especially among carriers of IL-6 polymorphisms associated with high production of IL-6 [3]. Consistent with this hypothesis are the results of a study conducted on patients with chronic hepatitis $C$, in which serum IL-6 levels predict development of HCC, but only among females, where they have a negative correlation with estradiol levels [4]. Nevertheless, others have observed that the association between high serum IL-6 concentrations and increased HCC risk depends on body mass index, and not gender [5].

In conclusion, while appreciating the attempt that Shakiba et al. made to give meta-analytical strength to the purported association between serum IL-6 and HCC, we wonder if further insight into this topic should not come from a re-analysis of the individual data of the studies they cite. This approach would allow them to take into account important modulating factors such as gender and body mass index.

\section{Disclosure}

Authors report no conflict of interest.

\section{References}

1. Shakiba E, Ramezani M, Sadeghi M. Evaluation of serum interleukin-6 levels in hepatocellular carcinoma patients: a systematic review and meta-analysis. Clin Exp Hepatol 2018; 4: 182-190.

2. Naugler WE, Sakurai T, Kim S, et al. Gender disparity in liver cancer due to sex differences in MyD88-dependent IL-6 production. Science 2007; 317: 121-124.

3. Falleti E, Fabris C, Toniutto P, et al. Interleukin-6 polymorphisms and gender: relationship with the occurrence of hepatocellular carcinoma in patients with end-stage liver disease. Oncology 2009; 77: 304-313.

4. Nakagawa H, Maea S, Yoshida H, et al. Serum IL-6 levels and the risk for hepatocarcinogenesis in chronic hepatitis $\mathrm{C}$ patients: an analysis based on gender differences. Int J Cancer 2009; 125: 2264-2269.

5. Ohishi W, Cologne JB, Fujiwara S, et al. Serum interleukin-6 associated with hepatocellular carcinoma risk: a nested case-control study. Int J Cancer 2014; 134: 154-163. 